\title{
The Use of Complementary and Alternative Medicine Among Chinese Women with Breast Cancer
}

\author{
Zhi Chen, M.D., Ph.D., ${ }^{1}$ Kai Gu, M.D., M.P.H., ${ }^{2}$ Ying Zheng, M.D., ${ }^{2}$ Wei Zheng, M.D., Ph.D., ${ }^{1}$ \\ Wei Lu, M.D., M.P.H., ${ }^{2}$ and Xiao Ou Shu, M.D., Ph.D. ${ }^{1}$
}

\begin{abstract}
Objectives: Using data from the Shanghai Breast Cancer Survival Study (SBCSS), we estimated the prevalence and perceived benefits of complementary and alternative medicine (CAM) and Chinese herbal medicine (CHM), and relevant demographic and clinical factors.

Design: This was a cross-sectional cohort study.

Subjects/setting/location: Subjects were 5046 women who participated in the SBCSS, conducted in urban Shanghai, China.

Interventions/outcome measures: Data on CAM use (at least once a week for at least 1 month), patient sociodemographics, and medical history were collected using a structured questionnaire.

Results: The average diagnosis age of participants was 53.5 years. Interviews were completed about 6.5 months after diagnosis. Ninety-seven percent (97\%) of participants used CAM therapy after diagnosis. Supplements were the most common type, followed by $\mathrm{CHM}$, and physical activity. Walking was the most popular type of physical activity. Almost all CHM users used CHM as part of their cancer treatment; three quarters reported $\mathrm{CHM}$ use for boosting the immune system. About two thirds of women considered CHM effective. Supplement users were more likely to have higher income, higher educational attainment, be married, have undergone or completed radiotherapy, used tamoxifen, have estrogen receptor (ER)-negative/progesterone receptor (PR)-negative cancer, and have menopausal symptoms. CHM use was associated with younger age, higher income, menopausal symptoms, completion of chemotherapy, and past tamoxifen use. Patients with an earlier clinical stage or who had undergone radiotherapy used less CHM. Chemotherapy or radiotherapy and cancer metastasis were positively related to physical activity participation. Current tamoxifen use, ER-/PR- cancer, higher educational attainment, and reporting average or better quality of life were inversely related to exercise participation.

Conclusions: Given the high prevalence of CAM use in patients with breast cancer and the variety of types of CAM, more research is needed to determine the impact of CAM's effectiveness and safety and interaction with conventional cancer treatment on breast cancer survival.
\end{abstract}

\section{Introduction}

Tomplementary and alternative medicine (CAM) is defined as therapies that are not typically offered within conventional health care settings, and include biologic, mind-body, energy, and spiritual therapies. The most common types of CAM include ingested therapies such as herbs and vitamins, homeopathic remedies, and Traditional Chinese Medicine (TCM), as well as psychologic, physical, and spiritual techniques. ${ }^{1-3}$ CAM is a growing field in health care, particularly among patients with cancer. CAM use is common among patients with breast cancer, and its prevalence varies from $16 \%$ to $84 \%{ }^{4-11}$

${ }^{1}$ Vanderbilt Epidemiology Center, Department of Medicine, Vanderbilt School of Medicine and Vanderbilt-Ingram Cancer Center, Nashville, TN.

${ }^{2}$ Department of Cancer Control and Prevention, Shanghai Municipal Center for Disease Control and Prevention, Shanghai Institute of Preventive Medicine, Shanghai, China. 
Previous studies on the utilization of CAM among patients with cancer have been conducted predominantly in North America and Western Europe countries. ${ }^{12-14}$ To our knowledge, only 1 report, which is from our group, has systematically described CAM use among Chinese women who survived 4.3 years after diagnosis of breast cancer. ${ }^{15}$ Using data from a population-based cohort study of 5046 patients with breast cancer, we estimated the prevalence and perceived benefits of CAM and Chinese herbal medicine (CHM) use and related demographic and clinical factors among Chinese women with breast cancer who were interviewed an average of 6 months after cancer diagnosis.

\section{Materials and Methods}

Study subjects were women who were diagnosed with a primary breast cancer and were enrolled in the Shanghai Breast Cancer Survival Study, a population-based, epidemiologic study of breast cancer survivors in Shanghai, China. All study subjects were permanent residents of urban Shanghai who were 20-75 years of age, newly diagnosed with breast cancer between April 1, 2002 and December 31, 2006, and alive at the time of interview. Study enrollment took place about 6 months after cancer diagnosis. Through the population-based Shanghai Cancer Registry, we identified 6303 eligible women. Of these, 5046 women participated in the current study, a response rate of $80.1 \%$. Nonparticipation was mainly due to refusal (755 cases; $12.0 \%)$, moving (168 cases; $2.7 \%$ ), out of town (90 cases; $1.4 \%$ ), inability to locate potential subjects ( 80 cases; $1.3 \%$ ), and other miscellaneous reasons (164 cases; $2.6 \%$ ).

Detailed information on the use of CAM among the study participants was elicited via an in-person interview using a structured questionnaire conducted by trained interviewers. CAM users were defined as women who used at least 1 type of CAM therapy once a week for 1 month or longer after they were diagnosed with breast cancer. Types of CAM included in this study are TCM, (which encompasses both CHM and acupuncture), supplements (e.g., ginseng, omega3 fatty acid, shark cartilage, honey tonic, melatonin, vitamins, etc.), physical activity (walking, running, gymnastics, body building on apparatus, qigong, t'ai chi, jogging, dancing, etc.), and participation in a support group. Participants were asked about duration of use for each type of CAM. TCM users were also questioned about indications for use and perceived effectiveness.

Participant sociodemographic and medical-related information was also collected during the in-person interview. This included age at diagnosis, marital status, annual household income, educational level, age at menarche and menopause, chemotherapy, radiotherapy, tamoxifen use, and whether the participant had undergone surgery. Anthropometrics, including weight, height, and waist and hip circumferences, were taken according to a standard protocol. In addition, medical charts were reviewed to obtain information on treatment, such as estrogen receptor (ER) and progesterone receptor (PR) status and cancer stage. The study was approved by the institutional review boards of all institutions involved in the study, and written, informed consent was obtained from all participants prior to interview.

All data analyses were performed by using Statistical Analysis Software (SAS, version 9.1, SAS Institute, Cary,
Table 1. Demographic and Clinical Characteristics of the Shanghai Breast Cancer Survival Study, 2002-2006

\begin{tabular}{cc}
\hline & $\begin{array}{c}\text { Prevalence among patients } \\
\text { with breast cancer }\end{array}$ \\
Characteristic & $(\mathrm{N}=5046)$ \\
\hline
\end{tabular}
Age at diagnosis (years) 40-49

$50-59$

$\geq 60$

2006

1492

1305

4.8

Menopausal status

Premenopausal

Postmenopausal

2467

2579

Yes

3604

1442

No

2342

1898

$<$ High school

High school

$>$ High school

806

Household income

(yuan/year)

$<8,400$

$8,400-<12,000$

1406

1489

$\geq 12,000$

2151

27.8

Marital status

Married

Unmarried

4437

609

ER/PR status

$\mathrm{ER}+/ \mathrm{PR}+$

ER-/PR-

Mixed

Unknown

Stage (TNM)

0 , I

IIa

$\mathrm{IIb}$

III, IV

Missing

2489

1406

949

202

1837

1646

837

489

237

Radiotherapy

Yes

Current

Complete

No

Chemotherapy

Yes

Current

Complete

Unknown

No

Tamoxifen use

Yes

Current

Complete

No

Unknown

Metastasis

Yes

No

Unknown

405

1213

3428

8.0

24.0

67.9

676

13.4

3920

77.7

$<0.1$

449

8.9

Perceived overall quality

of life

Poor or very poor

Average

418

1011

1609

2423

3

20.0

31.9

48.0

$52 \quad 1.0$

4937

97.8

57

Good or very good

3687

8.3

$\mathrm{ER}$, estrogen receptor; $\mathrm{PR}$, progesterone receptor; $\mathrm{TNM}$, primary tumor, regional lymph nodes, and distant metastasis. 
Table 2. Types and Prevalence of Complementary and Alternative Medicine (CAM) Therapies (Shanghai Breast Cancer Survival Study, 2002-2006)

\begin{tabular}{lcc}
\hline Types of CAM therapies used & Cases (N) & Prevalence of use (\%) \\
\hline CAM overall & 4906 & 97.2 \\
Any supplements & 3896 & 77.2 \\
Ganoderma lucidum capsules, sporophyte & 2947 & 58.4 \\
Vitamins & 1850 & 36.7 \\
All types of ginseng or its products & 726 & 14.4 \\
Deep ocean fish oil & 140 & 2.8 \\
Lecithin & 66 & 1.3 \\
Active fungus products (Angli \#1, etc.) & 53 & 1.1 \\
Shark cartilage capsules & 46 & 0.9 \\
Melatonin & 28 & 0.6 \\
Royal jelly & 23 & 0.5 \\
Otherb & 3600 & 71.2 \\
TCM & 3878 & 76.9 \\
CHM & 3873 & 76.8 \\
Acupuncture & 20 & 0.4 \\
\hline
\end{tabular}

${ }^{\mathrm{a} A}$ product similar to honey.

bIncluding coenzyme Q10 capsules, turtle pills, and miscellaneous unspecified supplements.

TCM, Traditional Chinese Medicine; CHM, Chinese Herbal medicine.

NC). Types and prevalences of CAM therapies used, indications for use, and perceived effectiveness of TCM treatments were described with percentages. The $\chi^{2}$ test was applied for categorical comparison of data. Logistic analyses were conducted to evaluate factors that correlated with supplement use, CHM use, and participation in physical activity. The significance level of all analyses was set at $\alpha=0.05$, and all tests were two-sided.

\section{Results}

The sociodemographic and medical characteristics of study participants are shown in Table 1 . The average age at cancer diagnosis was 53.5 years. About 51\% were postmenopausal and $71.4 \%$ reported having menopausal symptoms. More than half (53.6\%) had attained at least a high school education. Forty-two percent (42\%) had a yearly household income of more than 12,000 yuan/year, $28 \%$ reported less than 8,400 yuan/year, and about 30\% reported income between 8,400 and 12,000 yuan/year. One (1) yuan was approximately equal to 0.12 U.S. dollars during our study recruitment period. The vast majority of study participants $(87.9 \%)$ were married and living with a spouse at the time of the study enrollment. Almost all participants (99.8\%) had undergone surgery for breast cancer. Approximately $49.3 \%$ were $\mathrm{ER}+/ \mathrm{PR}+, 27.9 \%$ were $\mathrm{ER}-/ \mathrm{PR}-, 12.3 \%$ were
$\mathrm{ER}+/ \mathrm{PR}-$, and $6.5 \%$ were ER-/PR+. Approximately $4 \%$ were missing information on ER/PR status. About $95 \%$ had cancer staging data, of which $85.6 \%$ were stage $0-$ II. About $78 \%$ had completed chemotherapy and $13.4 \%$ were still on adjuvant chemotherapy at enrollment, while $24.0 \%$ had completed radiotherapy and $8.0 \%$ were currently undergoing radiotherapy. For tamoxifen use, 31.9\% reported previous use and $20.0 \%$ current use. Fifty-two (52) participants had metastatic cancer at enrollment. Nineteen percent (19\%) reported good or very good quality of life, $73 \%$ were satisfied with their quality of life, and $8 \%$ reported poor or very poor quality of life.

Table 2 presents the prevalence of CAM therapy use. Almost all study participants $(97.2 \%)$ reported previous or current use of at least 1 type of CAM therapy after being diagnosed with breast cancer. Supplements (77.2\%) were the most commonly used type of CAM. The most commonly used supplements were Ganoderma lucidum (lingzhi) capsules and sporophyte $(58.4 \%)$, followed by vitamins $(36.7 \%)$, and all types of ginseng or ginseng products $(14.4 \%)$. The vast majority of ginseng users $(14.3 \%)$ used American or white ginseng. About three fourths of participants reported using TCM. CHM was the dominant type of TCM used $(76.8 \%)$, while acupuncture use was rare $(0.4 \%)$.

Table 3 presents the perceived effectiveness of CHM by indications for use. Of participants who used CHM to assist

Table 3. Perceived Effectiveness of Chinese Herbal Medicine (CHM) Use by Indication for Use (Shanghai Breast Cancer Survival Study, 2002-2006)

\begin{tabular}{|c|c|c|c|c|c|}
\hline \multirow[b]{2}{*}{ Indicataion } & \multirow{2}{*}{$\frac{\text { CHM user }}{\%}$} & \multicolumn{4}{|c|}{ Perceived effectiveness (\%) } \\
\hline & & Very effective & Effective & Ineffective & Unknown \\
\hline 1. Treating cancer & 95.2 & 4.3 & 51.6 & 2.3 & 41.8 \\
\hline 2. Boosting immune system & 75.7 & 3.7 & 55.9 & 2.4 & 38.0 \\
\hline 3. Lessening menopausal symptoms & 29.4 & 4.0 & 58.4 & 2.6 & 35.0 \\
\hline 4. Other & 21.3 & 10.5 & 64.2 & 2.1 & 23.2 \\
\hline
\end{tabular}


with cancer treatments, about $4 \%$ perceived $\mathrm{CHM}$ as very effective, $52 \%$ as effective, while $2.3 \%$ considered $\mathrm{CHM}$ ineffective, and $41.8 \%$ reported the effectiveness as unknown. The percentage of those who reported CHM as very effective or effective for boosting the immune system or lessening menopausal symptoms varied between 59\% and $62 \%$.

The most common types of physical activity and their prevalences are described in Table 4. Two thirds of study participants $(64.5 \%)$ reported regular participation in physical activity, and $1.8 \%$ of patients regularly attended support groups. The most prevalent type of physical activity in our study population was walking $(70.6 \%)$, followed by gymnastics, body building and dancing $(35.8 \%)$, and traditional Chinese exercises including qigong, Chinese t'ai chi boxing, and Mulan boxing (9.1\%). Approximately 16\% reported sweating every time they exercised, while $68.6 \%$ reported not sweating at all. Forty-three percent (43.0\%), 28.6\%, and $28.4 \%$ reported exercising less than 2 hours, 2-4 hours, and greater than 4 hours per week, respectively. Exercise intensity was estimated using standard metabolic equivalent (MET) values. ${ }^{16}$ Approximately $15 \%$ of study participants exercised more than 22 MET-hours per week.

We further evaluated factors related to supplement use, CHM use, and regular participation in physical activity (Table 5). Supplement use was associated with having menopausal symptoms [odds ratios $(\mathrm{OR})=1.38$ : $95 \%$ confidence interval $(\mathrm{CI})=1.19-1.61$, educational attainment $(\mathrm{OR}=1.41$ : 95\% CI 1.20-1.64 for high school level and OR = 2.00: 95\% CI 1.57-2.56 for higher than high school level), high household income $(\mathrm{OR}=1.27$ : $95 \% \mathrm{CI} 1.06-1.51$ for 8,400 yuan to 12,000 yuan/year and $\mathrm{OR}=1.81$ : 95\% CI 1.51-2.17 for greater than 12,000 yuan/year), being married $(\mathrm{OR}=1.33$ : 95\% CI 1.09-1.62), having undergone $(\mathrm{OR}=1.34$ : $95 \% \mathrm{CI}$
1.02-1.77) or completed radiotherapy ( $\mathrm{OR}=1.31: 95 \% \mathrm{CI}$ 1.09-1.56), and current tamoxifen use (OR $=1.36$ : $95 \% \mathrm{CI}$ 1.12-1.66). CHM use was more common among participants aged $40-49$ years $(\mathrm{OR}=1.40: 95 \% \mathrm{CI}=1.01-1.93)$, those who reported presence of menopausal symptoms, high family income, completed adjuvant chemotherapy (OR $=1.70: 95 \%$ CI 1.34-2.15), and former tamoxifen use (OR $=1.29$ : 95\% CI 1.09-1.53). Participants were less likely to use CHM if they were currently undergoing radiotherapy $(\mathrm{OR}=0.61: 95 \% \mathrm{CI}$ $0.47-0.78$ ) or had stage IIa and IIb cancer (OR $=0.82: 95 \%$ CI 0.69-0.97 and OR =0.81: 95\% CI 0.66-0.99, respectively). Regular participation in physical activity was more common among participants who had received or were currently undergoing adjuvant chemotherapy, radiotherapy, or had cancer metastasis. Participants were less likely to regularly participate in physical activity if they had higher educational attainment $(\mathrm{OR}=0.75$ : 95\% CI 0.62-0.91), had ER-/PR- cancer $(\mathrm{OR}=0.84: 95 \% \mathrm{CI} 0.72-0.98)$, were currently taking tamoxifen (OR $=0.65$ : 95\% CI 0.55-0.77), or perceived average or better quality of life (OR $=0.66$ : $95 \%$ CI $0.54-0.81$ and $\mathrm{OR}=0.49: 95 \%$ CI $0.38-0.63$, respectively).

\section{Discussion}

The prevalence of CAM use among the Chinese women with breast cancer in our study is higher (97.2\%) than that reported among patients with breast cancer in the United States (84\%). ${ }^{9}$ Supplements were the most commonly used type of CAM, followed by CHM. This finding differs from our previous report, which indicated that CHM was the most commonly used type of CAM in this population of Chinese patients with breast cancer. ${ }^{15}$ This difference is due to the fact that our earlier study did not include vitamins in the cat-

Table 4. Prevalence of Regular Physical Activity (Shanghai Breast Cancer Survival Study, 2002-2006)

\begin{tabular}{|c|c|c|}
\hline Exercises & Cases (N) & Prevalence (\%) \\
\hline Regular participation in physical activity & 3256 & 64.5 \\
\hline Support group attendance & 91 & 1.8 \\
\hline \multicolumn{3}{|l|}{ Exercise type $\mathrm{a}^{\mathrm{a}}$} \\
\hline Walking, walking on oval-shaped pebbles & 2298 & 70.6 \\
\hline Gymnastics, body building, dancing & 1164 & 35.8 \\
\hline $\begin{array}{l}\text { Qigong, Chinese t'ai chi boxing, } \\
\text { Mulan boxing, kicking the shuttlecock }\end{array}$ & 297 & 9.1 \\
\hline $\begin{array}{l}\text { Jogging, swimming, rope skipping, stair } \\
\text { Climbing }\end{array}$ & 70 & 1.4 \\
\hline Other & 681 & 20.9 \\
\hline \multicolumn{3}{|l|}{ Sweating during exercise $\mathrm{e}^{\mathrm{a}}$} \\
\hline Usually did not sweat & 2232 & 68.6 \\
\hline Sweat most of the time & 489 & 15.0 \\
\hline Sweat every time & 535 & 16.4 \\
\hline \multicolumn{3}{|l|}{ Average exercise hours/week ${ }^{a}$} \\
\hline$>0-<2$ & 1396 & 43.0 \\
\hline $2-<4$ & 929 & 28.6 \\
\hline$\geq 4$ & 923 & 28.4 \\
\hline \multicolumn{3}{|l|}{ Average MET-h/week ${ }^{a}$} \\
\hline$>0-21$ & 2778 & 85.5 \\
\hline $22-31$ & 287 & 8.8 \\
\hline$\geq 32$ & 183 & 5.6 \\
\hline
\end{tabular}

${ }^{\mathrm{a} A m o n g}$ exercisers only.

MET, metabolic equivalent. 
Table 5. Relationship Between Complementary and Alternative Medicine Use, Regular Physical Activity and Demographic and Clinical Characteristics (Shanghai Breast Cancer Survival Study, 2002-2006)

\begin{tabular}{|c|c|c|c|}
\hline Variables & $\begin{array}{c}\text { Supplement use } \\
\text { OR }^{\mathrm{a}}(95 \% \mathrm{CI})\end{array}$ & $\begin{array}{l}\text { Any CHM use } \\
\text { OR }^{\mathrm{a}}(95 \% \mathrm{CI})\end{array}$ & $\begin{array}{c}\text { Any regular physical } \\
\text { activity } \\
\text { OR }{ }^{\mathrm{a}}(95 \% \mathrm{CI})\end{array}$ \\
\hline \multicolumn{4}{|l|}{ Age at diagnosis (years) } \\
\hline$<40$ & 1.00 & 1.00 & 1.00 \\
\hline $40-49$ & $1.33(0.94-1.87)$ & $1.40(1.01-1.93)$ & 1.03 (0.77-1.39) \\
\hline $50-59$ & 1.35 (0.92-1.99) & $1.43(0.99-2.07)$ & $0.92(0.67-1.28)$ \\
\hline$\geq 60$ & $1.35(0.89-2.05)$ & $1.07(0.72-1.59)$ & $0.99(0.69-1.41)$ \\
\hline \multicolumn{4}{|l|}{ Menopausal status } \\
\hline Premenopausal & 1.00 & 1.00 & 1.00 \\
\hline Postmenopausal & $0.85(0.69-1.06)$ & $0.83(0.66-1.03)$ & $1.06(0.88-1.27)$ \\
\hline \multicolumn{4}{|l|}{ Menopausal symptoms } \\
\hline No & 1.00 & 1.00 & 1.00 \\
\hline Yes & 1.38 (1.19-1.61) & 1.37 (1.18-1.59) & $0.92(0.81-1.06)$ \\
\hline \multicolumn{4}{|l|}{ Education } \\
\hline$<$ High school & 1.00 & 1.00 & 1.00 \\
\hline$=$ High school & $1.41(1.20-1.64)$ & $1.01(0.87-1.18)$ & $0.89(0.78-1.02)$ \\
\hline$>$ High school & $2.00(1.57-2.56)$ & $1.02(0.82-1.26)$ & $0.75(0.62-0.91)$ \\
\hline \multicolumn{4}{|l|}{$\begin{array}{l}\text { Household income } \\
\text { (vuan/year) }\end{array}$} \\
\hline $\begin{array}{l}\text { (yuan/year) } \\
<8,400\end{array}$ & 1.00 & 1.00 & 1.00 \\
\hline $8,400-<12,000$ & $1.27(1.06-1.51)$ & $1.16(0.96-1.39)$ & $1.14(0.97-1.34)$ \\
\hline$\geq 12,000$ & $1.81(1.51-2.17)$ & $1.25(1.04-1.50)$ & $1.06(0.90-1.24)$ \\
\hline \multicolumn{4}{|l|}{ Marital status } \\
\hline Unmarried & 1.00 & 1.00 & 1.00 \\
\hline Married & 1.33 (1.09-1.62) & $1.22(0.99-1.49)$ & $1.02(0.85-1.23)$ \\
\hline \multicolumn{4}{|l|}{ ER/PR status } \\
\hline $\mathrm{ER}+/ \mathrm{PR}+$ & 1.00 & 1.00 & 1.00 \\
\hline $\mathrm{ER}-/ \mathrm{PR}-$ & $1.26(1.05-1.51)$ & $1.18(0.98-1.41)$ & $0.84(0.72-0.98)$ \\
\hline Mixed & $1.07(0.89-1.29)$ & $1.10(0.92-1.33)$ & $0.91(0.77-1.06)$ \\
\hline Unknown & $1.04(0.74-1.46)$ & $0.53(0.39-0.73)$ & $1.02(0.75-1.39)$ \\
\hline \multicolumn{4}{|l|}{ Stage (TNM) } \\
\hline $0, \mathrm{I}$ & 1.00 & 1.00 & 1.00 \\
\hline IIa & $0.92(0.78-1.08)$ & $0.82(0.69-0.97)$ & $1.06(0.91-1.22)$ \\
\hline $\mathrm{IIlb}$ & $0.89(0.72-1.10)$ & $0.81(0.66-0.99)$ & $1.06(0.88-1.27)$ \\
\hline III, IV & $0.81(0.63-1.05)$ & $0.87(0.68-1.13)$ & $1.16(0.93-1.45)$ \\
\hline Missing & $0.77(0.55-1.06)$ & $0.95(0.68-1.32)$ & $1.04(0.78-1.40)$ \\
\hline \multicolumn{4}{|l|}{ Chemotherapy } \\
\hline No & 1.00 & 1.00 & 1.00 \\
\hline \multicolumn{4}{|l|}{ Yes } \\
\hline Current & $1.26(0.94-1.69)$ & $0.89(0.67-1.17)$ & $1.69(1.29-2.21)$ \\
\hline Completed & $1.19(0.94-1.52)$ & $1.70(1.34-2.15)$ & $1.30(1.03-1.65)$ \\
\hline \multicolumn{4}{|l|}{ Radiotherapy } \\
\hline No & 1.00 & 1.00 & 1.00 \\
\hline \multicolumn{4}{|l|}{ Yes } \\
\hline Current & $1.34(1.02-1.77)$ & $0.61(0.47-0.78)$ & $1.41(1.12-1.76)$ \\
\hline Completed & $1.31(1.09-1.56)$ & $0.98(0.83-1.17)$ & $1.34(1.15-1.55)$ \\
\hline \multicolumn{4}{|l|}{ Metastasis } \\
\hline No & 1.00 & 1.00 & 1.00 \\
\hline Yes & $1.18(0.60-2.32)$ & $0.67(0.37-1.24)$ & $2.31(1.29-4.14)$ \\
\hline Unknown & $1.32(0.70-2.50)$ & $0.74(0.41-1.32)$ & $1.37(0.81-2.34)$ \\
\hline \multicolumn{4}{|l|}{ Tamoxifen use } \\
\hline No & 1.00 & 1.00 & 1.00 \\
\hline \multicolumn{4}{|l|}{ Yes } \\
\hline Current & $1.36(1.12-1.66)$ & $1.11(0.92-1.34)$ & $0.65(0.55-0.77)$ \\
\hline Completed & $1.06(0.89-1.26)$ & $1.29(1.09-1.53)$ & $0.88(0.76-1.02)$ \\
\hline \multicolumn{4}{|c|}{$\begin{array}{l}\text { Perceived overall quality } \\
\text { of life }\end{array}$} \\
\hline Poor or very poor & 1.00 & 1.00 & 1.00 \\
\hline Average & $1.10(0.87-1.40)$ & $1.11(0.87-1.41)$ & $0.66(0.54-0.81)$ \\
\hline Good or very good & $1.33(0.99-1.77)$ & $0.95(0.72-1.26)$ & $0.49(0.38-0.63)$ \\
\hline
\end{tabular}

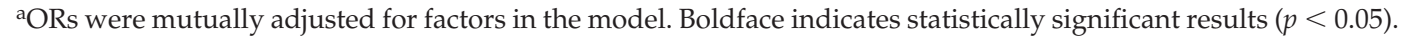

CHM, Chinese herbal medicine; CI, confidence interval; ER, estrogen receptor; OR, odds ratio; PR, progesterone receptor; TNM, primary tumor, regional lymph nodes, and distant metastasis. 
egory of supplements. The other major difference is that the current survey was conducted 6 months after cancer diagnosis, while our earlier study was conducted 4.3 years after cancer diagnosis.

Despite inconsistent reports on the benefits of $\mathrm{CHM}, \mathrm{CHM}$ use in the United States has continued to increase. ${ }^{17-19}$ The motivation for use and perceived effectiveness of CHM has rarely been reported. In our study, the main indications for CHM use were cancer treatment and boosting of the immune system, which is consistent with other reports. ${ }^{3,15,20}$ More than half of $\mathrm{CHM}$ users perceived $\mathrm{CHM}$ to be effective or very effective for their indications. This may explain the high prevalence of CHM use in our study population. The high rate of CAM use among Chinese breast cancer survivors raises safety concerns about possible adverse effects and drug interactions between conventional therapies and CAM therapies. For example, it has been suggested that antioxidants may reduce levels of the oxidizing free radicals created by radiotherapy and some forms of chemotherapy, thereby decreasing the effectiveness of these therapies. ${ }^{21-23}$ Abnormal liver function caused by use of alternative therapies during adjuvant chemotherapy for breast cancer has also been reported. ${ }^{24,25}$ In addition, heavy-metal contamination has been found in some herbal supplements, and these agents can act through a variety of mechanisms to alter the pharmacokinetic profile of concomitantly administered drugs. ${ }^{24}$ Our study findings call for more research in this area and emphasize the importance of encouraging communication between care providers and patients with breast cancer regarding their use of CAM therapies.

We found that approximately $65 \%$ of breast cancer survivors participated in regular exercise approximately 6 months after cancer diagnosis. For a subgroup of these study participants $(N=1448)$, precancer diagnosis exercise information was collected by a concurrent case-control study of breast cancer. The prediagnosis exercise participation rate in this subgroup was 30\%. These results suggest that breast cancer survivors in this Chinese population may be adopting a healthier lifestyle.

Predictors for supplement use, CHM use, and regular participation in physical activity in our study population were: younger age, menopausal symptoms, marital status, higher household income, chemotherapy, radiotherapy, tamoxifen treatment, and cancer metastasis. Supplement and CHM users were more likely to be younger, married, or have completed adjuvant chemotherapy and tamoxifen treatment, have menopausal symptoms, or higher household income than nonusers. This finding suggests that supplement and $\mathrm{CHM}$ use is correlated with socioeconomic status and users may have taken into consideration the potential interaction of CAM and conventional cancer treatment. Participation in physical activity was related to current chemotherapy or radiotherapy, recurrence/metastasis, and ER-positive breast cancer. These findings are consistent with previous reports. ${ }^{3,10,15,19}$ We also found that physical activity participation was inversely related to higher education, satisfaction with general quality of life, and taking tamoxifen. These results suggest that patients currently under treatment and those who had not completely recovered were actively seeking alternatives.

The current study has several strengths. First, our study is population-based, has a large sample size, and the re- sponse rate is high, all of which contributes to an unbiased estimation of CAM prevalence. Second, CAM use information was collected via an in-person interview using a detailed and structured questionnaire, which improved the quality of the information. Third, the survey was conducted during the period when patients had just completed or were near completion of conventional cancer treatments, which is important information for medical professionals providing conventional care to patients with breast cancer. Limitations of the study include a lack of detailed information on the composition of supplements and on the dietary assessments, which prohibited a comprehensive assessment of all sources of and specific types of vitamin intakes in our study.

\section{Conclusions}

In summary, CAM use among Chinese patients with breast cancer is high and concurrent with conventional cancer treatment. Given the concern about potential interactions between CAM therapies, particularly supplements, with chemotherapy and radiotherapy, and the limited amount of research conducted to date in this area, a systematic evaluation of the effectiveness and safety of CAM use is warranted. We are actively following up this cohort of patients with breast cancer, which will provide a unique opportunity for understanding the relationship between CAM use and breast cancer prognosis. In the meantime, our study results highlight the need for physicians and other health care providers to become better educated about CAM and communicate effectively with their patients with breast cancer regarding CAM use.

\section{Acknowledgments}

The authors are indebted to Dr. Fan Jin, the research team members, and most of all, the participants of the Shanghai Breast Cancer Survival Study for their contributions to this report. The authors have no conflicts of interest to declare. This study was supported by a research grant (DAMD17-021-0607) from the U.S. Department of Defense Breast Cancer Research Program. The U.S. Army Medical Research Acquisition Activity, 820 Chandler Street, Fort Detrick, MD 217015014 is the awarding and administering acquisition office. The content of the information does not necessarily reflect the position or the policy of the Government, and no official endorsement should be inferred.

\section{Author Disclosure Statement}

No competing financial interests exist.

\section{References}

1. National Center for Complementary and Alternative Medicine. What is complementary and alternative medicine? Online document at: http://nccam.nih.gov/health/whatiscam Accessed September 1, 2006.

2. Eisenberg DM, Kessler RC, Forster C, et al. Unconventional medicine in the United States; prevalence, costs and patterns of use. NEJM 1993;328:246-252.

3. Ashikage T, Boomer K, O’Brien P, Nelson L. Use of complementary and alternative medicine by breast cancer patients: Prevalence, patterns and communication with physicians. Supp Care Can 2002, 10:542-548. 
4. Crocetti E, Crotti N, Feltrin A, et al. The use of complementary therapies by breast cancer patients attending conventional treatment. Eur J Cancer 1998;34:324-328.

5. Rees R, Feigel I, Vickers A, et al. Prevalence of complementary therapy use by women with breast cancer: A population-based survey. Eur J Cancer 2000;36:1359-1364.

6. Moschen R, Kemmler G, Schweigkofler H, et al. Use of alternative/complementary therapy in breast cancer patients: A psychological perspective. Supp Care Cancer 2001;9: 267-274.

7. Boon $\mathrm{H}$, Stewart $\mathrm{M}$, Kennard MA, et al. Use of complementary/alternative medicine by breast cancer survivors in Ontario: Prevalence and perceptions. J Clin Oncol 2000;18: 2515-2521.

8. Ernst E, Casselith BR. The prevalence of complementary/alternative medicine in cancer: a systematic review. Cancer 1998;83:777-782.

9. Morris KT, Johnson N, Homer L, Walts D. A comparison of complementary therapy use between breast cancer patients and patients with other primary tumor sites. Am J Surg 2000;179:407-411.

10. Balneaves LG, Bottorff JL, Hislop TG, Herbert G. Levels of commitment: Exploring complementary therapy use by women with breast cancer. J Altern Complement Med 2006; 12:459-466.

11. Boon HS, Olatunde F, Zick SM. Trends in complementary/alternative medicine use by breast cancer survivors: comparing survey data from 1998 and 2005. BMC Women Health. 2007;7:4.

12. Burstein HJ, Gelber S, Guadagnoli E, Weeks JC. Use of alternative medicine by women with early-stage breast cancer. NEJM 1999;340:1733-1739.

13. DiGianni LM, Garber JE, Winer EP. Complementary and alternative medicine use among women with breast cancer. J Clin Oncol 2002;20:34S-38S.

14. Ribeiro MA, Harrigan RC. The use of complementary and alternative medicine by Asian women of Hawai'i in the treatment of breast cancer. Hawaii Med J 2006;65:198-205.

15. Cui Y, Shu XO, Gao Y, et al. Use of complementary and alternative medicine by Chinese women with breast cancer. Breast Cancer Res Treat 2004;85:263-270.
16. Ainsworth BE, Haskell WL, Whitt MC, et al. Compendium of physical activities: An update of activity codes and MET intensities. Med Sci Sports Exerc 2000;32:S498-S504.

17. Chang EY, Glissmeyer M, Tonnes $S$, et al. Outcomes of breast cancer in patients who use alternative therapies as primary treatment. Am J Surg 2006;192:471-473.

18. Jacobson JS, Workman SB, Kronenberg F. Research on complementary/alternative medicine for patients with breast cancer: A review of the biomedical literature. J Clin Oncol 2000;18:668-683.

19. Eisenberg DM, Davis RB, Ettner SL, et al. Trends in alternative medicine use in the United States, 1990-1997: Results of a follow-up national survey. JAMA 1998;280:1569-1575.

20. Astin JA, Reilly C, Perkins C, Child WL. Breast cancer patients' perspectives on and use of complementary and alternative medicine: A study by the Susan G. Komen Breast Cancer Foundation. J Soc Integr Oncol 2006;4:157-169.

21. D'Andrea GM. Use of antioxidants during chemotherapy and radiotherapy should be avoided. CA Cancer J Clin 2005;55:319-321.

22. Lamson DW, Brignall MS. Antioxidants in cancer therapy: Their actions and interactions with oncologic therapies. Altern Med Rev 1999;4:304-329.

23. Conklin KA. Dietary antioxidants during cancer chemotherapy: Impact on chemotherapeutic effectiveness and development of side effects. Nutr Cancer 2000;37:1-18.

24. Ahn JH, Kim SB, Yun MR, et al. Alternative therapy and abnormal liver function during adjuvant chemotherapy in breast cancer patients. J Korean Med Sci 2004;19:397-400.

25. De Smet PA. Herbal remedies. NEJM 2002;347:2046-2056.

Address reprint requests to:

Xiao Ou Shu, M.D., Ph.D.

Vanderbilt Epidemiology Center

Institute for Medicine \& Public Health

Vanderbilt University Medical Center

2525 West End Avenue, Suite 600

Nashoille, TN 37203-1738

E-mail: xiao-ou.shu@vanderbilt.edu 